\title{
Daycase cataract surgery
}

\author{
N P Strong, W Wigmore, S Smithson, S Rhodes, G Woodruff, A R Rosenthal
}

\begin{abstract}
The increasing demand for cataract surgery, combined with limited resources, has created renewed interest in daycase admission. We have audited the results of all daycase and inpatient cataract surgery in a large unit over a six-month period to determine the factors influencing daycase admission and surgical outcome; $34 \%$ of cataract patients were admitted as daycases in the study period. Neither distance travelled by the patient nor age appear to influence daycase admission. However, the differing policies of the individual consultant surgeons resulted in a wide variation in the number of operations performed on a daycase basis by each firm. The rate of preoperative complications was unaffected by daycase admission, by the grade of surgeon operating, or by the type of anaesthetic employed. Only $2 \cdot 1 \%$ of daycase admissions resulted in unplanned inpatient admission on the day of surgery, with another $2 \cdot 1 \%$ being readmitted within six months of surgery. Late cancellation of surgery was much lower for daycases $(0.4 \%)$ than for inpatients $(5 \cdot 1 \%)$.
\end{abstract}

Twenty years ago a patient undergoing cataract surgery might spend two weeks in hospital. ${ }^{12}$ In Britain an inpatient stay of only a few days is now usual, and in the United States $80 \%$ of cataract operations are now performed as daycases. ${ }^{3}$ Factors that have contributed to this change in management include an increasing demand for cataract surgery, ${ }^{4}$ the cost of extended inpatient care ${ }^{5}$ and a substantial drop in the number of elective surgical beds in the NHS. ${ }^{67}$

There have been several reports of daycase cataract surgery in the United Kingdom. ${ }^{589}$ One unit has set up a small dedicated day unit; ${ }^{5}$ all other reports concern patients managed in an inpatient ward. In the last year our department has elected to close an entire inpatient ward and set up a dedicated daycase unit. To analyse the impact of this major change we have audited the results of the first six months of operation of our daycase unit and have compared them with the inpatient ward in order to determine: (1) the factors influencing whether a patient is admitted as an inpatient or daycase; (2) the complication rate for daycases versus that for inpatients; (3) whether it is appropriate for junior surgeons (below consultant grade) to perform daycase surgery; (4) whether a significant number of unplanned inpatient admissions arise as a result of daycase surgery; and (5) the comparative rates of late cancellation of daycase and inpatient cataract surgery.

\section{Patients and methods}

The design of the ophthalmic daycase surgical unit (ODSU) and the medical and social criteria applied for patient selection for daycase cataract surgery are described elsewhere. ${ }^{10}$ The cataract surgery inpatients were managed on a separate but adjacent ward.

Cataract surgery was performed under either general or local anaesthesia. Daycase operations that required either general anaesthesia or local anaesthesia augmented with intravenous sedation were restricted to morning lists. Local anaesthesia was achieved with either retrobulbar or peribulbar infiltration, with or without oral or intravenous sedation.

\section{SURVEY DESIGN}

All patients admitted to the ODSU for cataract surgery over the first six months of its operation were entered on a database. All patients having cataract surgery as an inpatient during the same period were entered into a second database file. Patient data were obtained by examining the records of the ODSU, inpatient ward, operating theatre, and hospital computer. From these sources we obtained the patient's age, anaesthetic, consultant firm, surgeon operating and distance travelled to the hospital (derived from the postcode). In addition the individual hospital records of both inpatients and daycases were examined if $(a)$ the operation was cancelled, $(b)$ vitreous loss was noted in the threatre record. (c) an anterior chamber lens or no lens was implanted, $(d)$ the patient was readmitted.

Any patients undergoing further surgery at the same time as cataract extraction, such as a trabeculectomy or keratoplasty, were excluded from this study.

Statistical analysis was by two sample $t$ test $\chi^{2}$ test, and Fisher's exact test.

\section{Results}

Two hundred and thirty six patients underwent standard cataract surgery as daycases, accounting for $34 \%$ of all cataract operations performed during this period. One further patient was admitted, but the operation was cancelled. The proportion of all cataract patients managed as daycases rose during the period under study from $29 \%$ in the first two months to $32 \%$ in the second and $38 \%$ in the last two months.

In the six months of this study 487 patients were admitted as inpatients, of which 462 had the operation performed $(66 \%$ of all cataract operations) and 25 had surgery cancelled.

The daycase and inpatient cataract patients were compared to determine which factors might influence the decision to admit as an inpatient or daycase. Four variables were examined: the distance of the patient's home from the hospital; the patient's age; the type of anaesthetic used; and the identity of the surgical firm admitting the patient. 
Table 1 The percentage of all cataract operations carried out on a daycase basis by each of the consultant firms in the first six months of operation of the ODSU, and the relationship between the percentage of cataract surgery carried out under local anaesthesia and the percentage performed on a daycase basis for each of the consultant firms

\begin{tabular}{lll}
\hline $\begin{array}{l}\text { Consultant } \\
\text { no }\end{array}$ & $\begin{array}{l}\text { Percentage of cataracts } \\
\text { as daycases }\end{array}$ & $\begin{array}{l}\text { Percentage of cataracts } \\
\text { under local anaesthetic }\end{array}$ \\
\hline 1 & 23 & 50 \\
2 & 14 & 36 \\
3 & 33 & 32 \\
4 & 19 & 25 \\
5 & 74 & 90 \\
6 & 49 & 82 \\
7 & 35 & 65 \\
\hline
\end{tabular}

The distance that the patients travelled to hospital was very similar for the two groups $(\mathrm{p}=$ 0.43 ), and averaged 10.0 miles (SD 9.4) for daycases and 10.5 miles (SD 9.6) for inpatients. Similarly the age distribution of the two groups differed little $(p=0 \cdot 26)$, with an average age of 71.9 years $(S D 10.9)$ for the daycase patients and 72.9 years (SD 12.6) for the inpatients.

Daycase cataract surgery was more likely to be carried out under local anaesthesia $(90 \%$ of operations) than was the case for the inpatients $(56 \%)$. This difference is significant $\left(\mathrm{p}<10^{-6}\right)$.

Table 1 shows the percentage of cataract operations performed as daycase surgery by the different consultant firms. This varied from $14 \%$ to $74 \%$ of their total cataract workload, indicating a wide divergence in the surgeons' use of the daycase facility. Comparison of the different firms also showed a strong correlation between the proportion of cataract surgery carried out under local anaesthesia and the proportion of patients managed as daycases (Table 1 ).

A measure of the perioperative surgical complication rate was obtained by noting the number of cases of vitreous loss in each group. Table 2 shows vitreous loss rates for consultant and junior surgeons (that is, all surgeons below consultant grade) for daycases and inpatients. This shows that the junior surgeons performed more daycase surgery as a proportion of their total operative workload $(41 \%)$ than did the consultants $(26 \%)$. The rate of vitreous loss for daycases performed by juniors was slightly higher than for those done by consultants, but this difference was not significant $(p=0 \cdot 35)$, and for inpatients the consultant surgeons had the higher rate of vitreous loss, which again was not significant $(p=0 \cdot 14)$. Overall the rate of loss for all daycases $(4 \cdot 66 \%)$ did not differ significantly from that for inpatients $(4 \cdot 33 \%, p=0 \cdot 49)$.

The vitreous loss rate for all local anaesthetic cases was $4 \cdot 6 \%$ which was slightly higher than for general anaesthesia $(4 \cdot 2 \%)$, but not significantly $(p=0.97)$.

Unplanned admission to the inpatient ward may be due either to a decision not to discharge on the day of surgery or to a subsequent re-

Table 2 Vitreous loss rates for daycases and inpatients, according to grade of surgeon

\begin{tabular}{lcrl}
\hline & Consultants & \multicolumn{1}{l}{ funiors } & All surgeons \\
\hline Daycases & $3 / 90(3 \cdot 3 \%)$ & $8 / 146(5 \cdot 5 \%)$ & $11 / 236(4 \cdot 7 \%)$ \\
Inpatients & $14 / 255(5 \cdot 5 \%)$ & $6 / 207(2 \cdot 9 \%)$ & $20 / 462(4 \cdot 3 \%)$ \\
All patients & $17 / 345(4 \cdot 9 \%)$ & $14 / 353(4 \cdot 0 \%)$ & $31 / 698(4 \cdot 4 \%)$ \\
\hline
\end{tabular}

admission. Five patients were admitted as daycases but were then transferred to the inpatient ward $(2 \cdot 1 \%$ of all daycase cataract admissions). Two of these had been inappropriately accepted for daycase surgery despite not fulfilling the required criteria. Two others had vitreous loss during surgery and were kept in, and one was slow to recover from a general anaesthetic.

Another five daycase patients $(2 \cdot 1 \%)$ were readmitted at a later stage, all at least six or more days postoperatively. One developed endophthalmitis on the fifth postoperative day and was seen and admitted the following day. The second was admitted for repair of a small iris prolapse after a week. All the others developed late complications unrelated to daycase management. One fell and ruptured the cataract section three months postoperatively, and other patients were readmitted with retinal detachments three and five months postoperatively. No patient required immediate readmission or revision of surgery during the period under study.

Late cancellation of surgery is a major cause of wasted theatre time. Patients for daycase surgery are clerked in advance, so if a cause for cancellation or postponement of surgery is found there remains time to send for another patient. Consequently only one patient was admitted to the ODSU but then had surgery cancelled. This occurred because the notes had been mislaid $(0.4 \%$ of admissions). In the same period 25 patients were admitted to the inpatient ward for cataract extraction but had surgery cancelled ( $5 \cdot 1 \%$ of admissions). In two cases this was due to an infection contracted within a few days of the admission date and thus was unavoidable, and in three other cases there were problems with induction of local or general anaesthesia. However, for the other 20 patients $(4 \cdot 1 \%)$ the reason for cancellation was either that the patient had been inappropriately listed (seven patients) or was not medically fit for surgery (13 patients).

\section{Discussion}

When the ODSU was first opened the intention was to manage at least $25 \%$ of cataract admissions as daycases, but during the first six months the ODSU was used for $34 \%$ of all cataract surgery admissions, and this rate rose over the study period, indicating an increasing acceptance of daycase cataract surgery among patients and staff.

Our hospital draws patients from a large area, and it might have been expected that geographic factors would affect the decision whether to admit as a daycase; however our data show this was not the case. Some daycase patients travelled over 30 miles each way. Nor did the patients' ages appear to influence management, as age distribution was identical for the two groups.

Of the factors examined the one having the most influence on the decision to perform cataract surgery on inpatient or daycase basis was the policy of the consultant surgeon concerned. Daycase surgery accounted for $74 \%$ of the total number of cataract operations performed by one of the consultant firms, whereas another performed only $14 \%$ of cataract operations in this way. Furthermore the proportion of operations 
performed as daycase surgery by each firm was closely related to the proportion of cases operated on under local anaesthesia (Table 1). Although a general anaesthetic did not preclude daycase admission, the restriction of general anaesthesia to the morning lists meant that any firm that did a high proportion of its surgery under general anaesthesia was not likely to be able to do most of its surgery as daycases. In Britain there is a tradition of general anaesthetic cataract surgery which differs from that of many other countries, ${ }^{3}$ and which may have to be re-examined if daycase cataract surgery is to become more widespread.

The frequency of vitreous loss rate was adopted as a measure of peroperative surgical complications. This did not differ significantly between daycases and inpatients, even though a much higher proportion of the daycases were performed under local anaesthesia. Furthermore the vitreous loss rate for junior staff did not significantly differ from that of the consultant staff, and this would appear to justify the policy that all surgical grades should be allowed to undertake daycase surgery.

As the number of inpatient beds continues to decline it is important that unplanned overnight admissions are kept to a minimum. Of the 237 patients admitted to the ODSU for cataract surgery only five patients were kept in overnight afterwards $(2 \cdot 1 \%)$. Two of these were inappropriately listed for daycase surgery in the early days of the ODSU, and the listing procedure was amended to avoid repetition of these errors. Careful attention must be paid to patient selection for daycase cataract surgery, and it is essential that the patient understands the nature of the surgery and the type of anaesthetic that will be used. In addition adequate transport and social support must be available.

No patient required immediate readmission on being examined on the first postoperative day, and none required immediate revision of surgery. This is similar to the experience of Davies et al, and it contrasts with an earlier report of short-stay inpatient surgery in which a substantial proportion of cases were readmitted or not discharged, some of whom required early reoperation. ${ }^{11}$

An argument against daycase surgery is that the recognition, diagnosis, and treatment of immediate postoperative complications may be delayed. However, the earliest a patient was readmitted was the sixth day following surgery, owing to an endophthalmitis which had developed over the previous 24 hours. As $94 \%$ of inpatients were discharged by the end of the third postoperative day this patient would most probably have been discharged prior to developing this complication even if managed as an inpatient.

It has been suggested that it is not appropriate to perform daycase cataract surgery under general anaesthesia, ${ }^{12}$ and in previous reports daycase surgery has been performed exclusively under local anaesthesia ${ }^{8}{ }^{813}$ However, 24 cataract daycase operations $(10 \cdot 2 \%)$ were carried out under general anaesthesia during the study period as well as 156 other operations. ${ }^{10}$ Modern general anaesthesia now allows most patients to be fully ambulant on the day of surgery, and we believe that general anaesthesia as such is no longer a reason for inpatient management.
A subgroup of both inpatients and daycases having cataract surgery under local anaesthesia was studied in detail through the processes of admission, surgery, and follow-up and has been reported in preliminary form elsewhere (Rhodes $S$, proceedings of Age and Anaesthesia Meeting, Liverpool, April 1990). Among these patients it was found that there was no difference in patient satisfaction between inpatients and daycases, a finding in common with other studies (Lowe $\mathrm{K}$, proceedings of Second Annual Congress of the College of Ophthalmologists, Harrogate, 1990). $80 \%$ of patients stated that they would be happy to repeat the experience, and there was no difference in this respect between patients operated upon with or without sedation. Most of the patients who were not happy were upset by matters not specific to daycase surgery, such as the hardness of the operating table or the claustrophobic sensation of the operating drapes.

Last minute cancellation of operations, for whatever reason, can result in a substantial wastage of theatre time. Only one patient $(0 \cdot 4 \%)$ was admitted to the ODSU but did not have cataract surgery. Twenty five patients $(5 \cdot 1 \%)$ inpatients had their surgery cancelled. In 20 of these inpatients the cause for cancellation would have been detected if the patient had attended a preadmission assessment clinic, such as that attended by the daycase patients.

The results presented in this paper derive from an audit, and patients were not randomised between daycase and inpatient management. Nevertheless our experience indicates that daycase cataract surgery is not associated with an increase in morbidity, and that this is true for patients who were operated upon by junior as well as by consultant surgeons. It results in fewer late cancellations than inpatient admission, is acceptable to the patients, and can be performed under general anaesthesia provided suitable guidelines are followed.

We are grateful to J Sparrow, FCOphth, for statistical advice and C Nicholson for editorial assistance.

1 Strachan IM, Bowell RE. Reduced inpatient stay following cataract extraction. Trans Ophthalmol Soc UK 1972; 92: 62936.

2 Mills PV. Duration of inpatient care following cataract extraction. Trans Ophthalmol Soc UK 1972; 92: 637-43.

3 Severin SL. Outpatient cataract surgery. Semin Ophthalmol 1986; 1: 130-5.

4 Jay JL, Devlin ML. The increasing frequency of surgery for

cataract. Eye 1990; 4: 127-31.
Davies PD, Limacher E, Powell K. Outpatient cataract surgery 1982-1986. Eye 1987; 1: 728-34.

$6 \mathrm{Ogg}$ TW, Obey PA. The workload of a purpose built day surgical unit. Ann R Coll Surg Engl 1987; 69: 110-2.

$7 \mathrm{Ogg} \mathrm{TW}$, Heath P, Brownlie G. A case for the expansion of day Ogg TW, Heath P, Brownlie G. A case for the
surgery. Health Trends 1989; 21: 114-7.

8 Ingram RM, Banerjee D, Traynar MJ, Thompson RK. Daycase cataract surgery. Trans Ophthalmol Soc UK 1980; 100: 205-9.

9 Watts MT, Pearce JL. Day-case cataract surgery. $B r f$ Ophthalmol 1988; 72: 897-9.

10 Strong NP, Wigmore W, Rhodes S, Smithson B, Woodruff G, Rosenthal AR. An ophthalmic daycase unit. Health Trends in press.

11 Vernon SA, Cheng $H$. Comparison between the complications of cataract surgery following local anaesthesia with short stay and general anaesthesia with a five day hospitalisation. $\mathrm{BrF}$ Ophthalmol 1985; 69: 360-3.

12 Commission on the provision of surgical services. Guidelines for day case surgery. London: Royal College of Surgeons of England, 1985 .

13 Galin MA, Boniuk V, Obstbaum S, Glasser M. Outpatient cataract surgery. Trans Ophthalmol Soc UK 1975; 95: $42-5$. 